\title{
The Relationship Between Fat Tissue \& Lean Body Mass and Sit to Stand Task in Obese Individuals
}

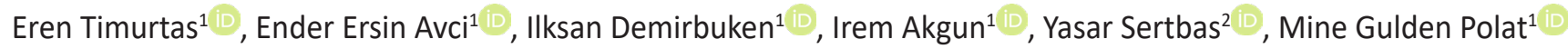 \\ ${ }^{1}$ Marmara University, Health Science Faculty, Department of Physiotherapy and Rehabilitation, Maltepe, Istanbul, Turkey \\ ${ }^{2}$ Department of Internal Medicine, Fatih Sultan Mehmet Education and Research Hospital, Küçükbakkalköy, Istanbul, Turkey \\ Correspondence Author: Irem Akgun \\ E-mail: iremm_akgun@hotmail.com
}

Received: $01.08 .2020 \quad$ Accepted: 29.05 .2021

\begin{abstract}
Objective: Obesity, currently one of the important health issues, can be defined through Fat Tissue Mass (FTM) and Lean Body Mass (LBM). The study aimed to investigate to what extent do FTM and LBM are associated with movement strategies of Sit-to-Stand (STS) task in individuals with obesity.

Methods: Forty-nine obese individuals $(52,83 \pm 7,39)$ with no diagnosis of any health condition included in the study. The Balance Master System was used to evaluate the STS movement. STS task was analyzed by means of Weight Transfer Time (WTT) (second), Rising Index (RI) (force exerted by legs-\%Body Weight), the Center of Gravity (COG) sway velocity (degrees per second). The FTM and LBM were regressed against each STS parameter including age as a covariate.

Results: The regression models could explain $10-21 \%$ of the variabilities in STS parameters: RI (21\%), COG sway velocity (11\%), WTT (10\%). FTM significantly related to RI $(\beta$ : $-0.287, p=0.040)$, but not with COG sway velocity $(\beta: 0.270, p=0.073)$ and WTT $(\beta:-0.038, p=0.802)$. LBM was significantly associated with RI ( $\beta$ : $0.435, p=0.003)$; yet, not with COG sway velocity ( $\beta:-0.100, p=0.066), W T T(\beta:-0.092, p=0.549)$.

Conclusion: This study revealed that FTM and LBM can explain the significant percent of the variation in RI during STS task, meaning a decrease in FTM and an increase in LBM provided support to rise during STS task. Moreover, an increase in FTM deteriorated postural stability. Improving LBM and decreasing FTM would be an effective strategy to improve STS in the obese population to increase their agility and could encourage physical activity participation.
\end{abstract}

Keywords: Obesity, Fat Tissue Mass, Lean Body Mass, Sit to Stand Task

\section{INTRODUCTION}

Obesity is currently becoming one of the most important public health issues. World Health Organization has stated that obesity had reached epidemic proportions globally (1). Furthermore, Finkelstein et al. reported an estimated obesity prevalence for the year 2030 through regression modeling and suggested that there will be an increase by $33 \%$ in obesity prevalence and $130 \%$ in severe obesity prevalence (2).

Parallel to the global increase in this health hazard, researches relating to various aspects of obesity has receiving a great deal of interest. However, functional limitations imposed by obesity and biomechanical alterations in daily functions have not been largely studied (3).

Existing literature proved that individuals with higher body weight have poor functional capacities. They also use altered strategies through functional tasks due to excessive and poorly disturbed fat tissue mass (4-6). The effect of higher Body Mass Index (BMI) including Lean Body Mass (LBM) and Fat Tissue Mass (FTM) on a higher level of functional limitations were first mentioned by Zioco et al. They indicated that lower LBM and higher FTM ratios increase the odds of functional limitations (7). The limited published studies in this field have mainly focused on the effect of obesity on plantar pressure distributions, postural balance and postural control during walking (3).

Changing a seated position to a standing, STS, is a fundamental movement for participation in many activities of daily living (8). A healthy individual performs STS movement approximately 60 times in a day (9). The ability to perform proper STS movement is considered to be an important determinant of functional fitness and independence. This task requires sufficient muscle strength to vertically accelerate body mass against the gravity and postural control ability from beginning to the end of the movement $(10,5,6)$. The increase in BMI considerably reduces trunk and lower extremity strengths, body power and ability to control postural stability (11). These functional disturbances may 
result in significant impairments in individuals' functional ability on sit-to-stand activity (STS).

If individuals are less capable of a rising body from a sitting to a standing position they might avoid performing STS movement as often (12). This behavior limits the participation of physically active tasks and encourages the sitting for long periods which would cause vigorous obesity and inactivity cycle.

Despite its importance in activities of daily living little research exist regarding the STS movement and its determinants in the obese population $(13,14)$. Furthermore, better knowledge of the implication of BMI by means of FTM and LBM on the STS movement of obese individuals would help us to develop proper interventions to improve their functional performance. It would also help to manage limited participation in physical activity indirectly by managing FTM and LBM. Therefore, the purpose of the current study was to investigate to what extent do FTM and LBM are associated with movement strategies of STS task in obese individuals.

\section{METHODS}

\subsection{Participants}

49 obese individuals (age: 52,83 \pm 7,39, BMI: 35,56 \pm $5,71)$ participated into the study. Inclusion criteria were (1) being at the age between 18-65; (2) BMI>30 kg/m²; (3) able to stand without support from a chair. Exclusion criteria were (1) cardiovascular weakness; (2) having neurological, metabolic, rheumatic or vestibular diseases; (3) injuries or previous surgery on the legs and no clinical knee and ankle instability; (3) having cognitive and behavioral problems. The participants were recruited from the Marmara University Department of Physiotherapy and Rehabilitation.

The study design was approved by the local ethical committee Marmara University Clinical Research Ethics Committee, Istanbul, Turkey (06.10.2017 - Protocol ID: 09.2017.604). All subjects were taken demographic features; age, height, weight and signed informed consent before participating in this study.

\subsection{Measurements}

\section{Body composition}

Body composition components which are BMI, LBM and FTM were measured without shoes and in light clothes by using a bioelectrical impedance analysis (BIA) based on a body composition analyzer (TANITA BC-418MA) (25).

\section{Sit to Stand Assessment}

The STS movement was evaluated by the Balance Master System (NeuroCom version 8.1, International, Inc.,
USA). The evaluation procedure for STS movement was completed according to the manufacturer's instructions (15).

All STS movements were performed on a long force platform comprising two force plates on barefoot. Force sensors located under the force platform measure the vertical forces exerted by the feet.

The measurements started from a seated position on an armless and backless chair, with arms resting by the sides. The participants were instructed to face the monitor while sitting, to keep both legs at shoulder width and to place their feet symmetrically and parallel to each other on the force platform. Participants were allowed to practice as much as trial they need to become familiar with the test procedure prior to data collection. They asked to follow the signs and react to them as quickly as possible. The test starts when the green "Go" sign appears on the monitor and participants were instructed to stand up as fast as possible without arm support. After a while, the monitor shows the "Hold Steady" sign. At this point, participants were asked to maintain their upright position. Steady standing lasts for 5 seconds to complete one STS trial. The system requires three STS trial and give a mean score obtained from these three trials for each parameter of STS.

STS task was investigated through some essential parameters such as weight transfer time, rising index, sway velocity and weight-bearing symmetry by the software of the system:

Weight transfer time (WTT), which is expressed in seconds $(s)$, is defined as the amount of time between the onset of the "Go" sign to move and the arrival of the center of gravity (COG) over the feet. Low scores are defined as good and high scores are bad. Rising index (RI) (expressed as a percent of body weight, \%) is the amount of force exerted by the legs during the rising phase of the STS movement. Insufficient lower extremity force will result in a failure to rise to a fully upright position. Low scores obtained from the rising index are considered as worse and high scores are good. Sway velocity, degrees per second, is the average amount of COG sway during the rise to stand and for the first five seconds following the rise. COG sway velocity during and immediately after the rise should be minimal. Therefore, low scores indicate good balance control and high scores are worse.

\subsection{Statistical Analysis}

Variance Inflation Factor (VIF) and regression correlation matrix for Klein Goldberger model were used to assess the collinearity among independent variables, after ShapiroWilk test and histograms were conducted to evaluate the normality of data.

Pearson correlation analysis was used for identifying association between Balance Master scores and independent variables. The characteristics of participants are provided by sex to enable meta-analyses and sample size calculations for future studies. The outcomes of interest, Balance Master 
scores were regressed against two independent variables: FTM and LBM. The regression analyses were mixed men and women as the distributions of key variables were not vastly different (23). All statistical analyses were performed using $R$ statistical. software (packages of 'olsrr'and 'lubridate' Version 3.6.0, St. Louis, Missouri, USA) was used (24).

\section{RESULTS}

The demographic and clinical characteristics of the subjects were illustrated in Table 1. There were significant correlations between FTM, LBM and STS parameters (RI, WTT and COG). There was a strong negative relationship between FTM and RI ( $r$ : $-0.423, p=0.047)$. Also, the correlation between LBM and $\mathrm{RI}$ are significantly positive ( $r$ : $0.628, p=0.028)$. The relationships between FTM and COG ( $r: 0.343, p=0.102)$, FTM and WTT ( $r:-.288, p=0.141)$, LBM and COG $(r:-0.136$, $p=0.216)$, LBM and WTT $(r:-0.330, p=0.119)$ were not statistically significance (Table 2 ).

Table 1. Demographic and clinical characteristics of the subjects

\begin{tabular}{|l|c|c|c|}
\hline \multirow{2}{*}{ Sex } & \multicolumn{2}{|c|}{ Subjects (n=49) } & \\
& $\begin{array}{c}\text { Male: } \mathbf{1 8} \\
(\% \mathbf{3 6 , 7 4 )}\end{array}$ & $\begin{array}{c}\text { Female: } \mathbf{3 1} \\
(\mathbf{\%} \mathbf{6 3 , 2 6 )}\end{array}$ & All Subjects \\
\hline Age (years) & $53.38 \pm 5.83$ & $52.61 \pm 8.10$ & $52.83 \pm 7.39$ \\
\hline Weight (kg) & $94.38 \pm 9.41$ & $92.31 \pm 20.07$ & $94.52 \pm 16.77$ \\
\hline Height (cm) & $173.33 \pm 6.30$ & $159.35 \pm 5.32$ & $164.22 \pm 8.57$ \\
\hline BMI (kg/m²) & $31.38 \pm 2.54$ & $36.84 \pm 6.83$ & $35.56 \pm 5.71$ \\
\hline FTM (kg) & $24.88 \pm 4.83$ & $39.29 \pm 12.85$ & $34.68 \pm 12.54$ \\
\hline LBM (kg) & $69.45 \pm 6.44$ & $53.54 \pm 7.62$ & $59.15 \pm 10.47$ \\
\hline STS-WTT (sec) & $0.47 \pm 0.30$ & $0.45 \pm 0.21$ & $0.44 \pm 0.22$ \\
\hline STS-RI (\% Wt) & $24.17 \pm 10.64$ & $18.90 \pm 6.31$ & $21 \pm 8.68$ \\
\hline $\begin{array}{l}\text { STS-COG sway } \\
\text { velocity (deg/sec) }\end{array}$ & $4.11 \pm 1.25$ & $4.27 \pm 1.32$ & $4.25 \pm 1.25$ \\
\hline
\end{tabular}

FTM: Fat Tissue Mass, LBM: Lean Body Mass, STS: Sit to Stand, WTT: Weight Transfer Time, RI: Rising Index, COG: Center of Gravity, Wt: Weight

Table 2. Correlation analysis between each predictor and balance master scores indicators

\begin{tabular}{|c|c|c|c|c|c|c|}
\hline & \multicolumn{4}{|c|}{ OUTCOMES (BALANCE MASTER SCORES) } \\
\hline Predictors (range) & \multicolumn{2}{|c|}{ RI } & \multicolumn{2}{|c|}{ COG } & \multicolumn{2}{c|}{ WTT } \\
\hline FTM & -0.423 & $\mathbf{0 . 0 4 7 *}$ & 0.343 & 0.102 & -0.288 & 0.141 \\
\hline LBM & 0.628 & $\mathbf{0 . 0 2 8 *}$ & -0.136 & 0.216 & -0.330 & 0.119 \\
\hline
\end{tabular}

FTM: Fat Tissue Mass, LBM: Lean Body Mass, COG: Center of Gravity, WTT: Weight Transfer Time, ${ }^{*} p<0,05$ (statistically significant)

Overall, the regression models could explain $10-21 \%$ of the variabilities in STS parameters: rising index (21\%), COG sway velocity $(11 \%)$, the weight transfer time (10\%). FTM was significantly associated with rising index $(\beta:-0.287$, $p=0.040)$, but not with COG sway velocity $(\beta: 0.270, p=0.073)$ and weight transfer time $(\beta$ : $-0.038, p=0.802)$. LBM was significantly related to rising index $(\beta: 0.435, p=0.003)$; yet, not with COG sway velocity $(\beta$ : $-0.100, p=0.066)$, weight transfer time $(\beta:-0.092, p=0.549)$. The results of regression analyses are presented in Table 3.
Table 3. Multiple regression analysis: relationship between each predictor and balance master scores indicators

\begin{tabular}{|l|l|l|l|l|l|l|}
\hline \multicolumn{5}{|c|}{ OUTCOMES (BALANCE MASTER SCORES) } \\
\hline & RI & \multicolumn{3}{l|}{ COG } & \multicolumn{1}{l|}{ WTT } \\
\hline Predictors (range) & $\beta$ & $p$ & $\beta$ & $p$ & $\beta$ & $p$ \\
\hline FTM & -0.287 & $\mathbf{0 . 0 4 0 *}$ & 0.270 & 0.073 & -0.038 & 0.802 \\
\hline LBM & 0.435 & $\mathbf{0 , 0 0 3 *}$ & -0.100 & 0.066 & $-0,092$ & 0.549 \\
\hline$R^{2}$ & $\mathbf{0 . 2 1}$ & $\mathbf{0 . 1 1}$ & & $\mathbf{0 . 1 0}$ & \\
\hline
\end{tabular}

FTM: Fat Tissue Mass, LBM: Lean Body Mass, COG: Center of Gravity, WTT: Weight Transfer Time, $R$ : Regression, ${ }^{*} p<0,05$ (statistically significant)

\section{DISCUSSION}

The aim of the current study was to investigate to what extent FTM and LBM are associated with movement strategies of STS task in individuals with obesity. Up to date, few studies investigating the STS task in obese individuals have been reported in the scientific literature, although it is the most frequently used task in daily living (16).

The results of the current study revealed that FTM and LBM scores have a significant role in the STS task for individuals with obesity. The main finding of the current study was the observed relationship between the rising index with both FTM and LBM values. Rising index was found to be significantly related with FTM negatively and LBM positively. It shows that higher and lower rising indexes seen in obese individuals were a consequence of higher LBM and FTM, respectively. Since the rising index is considered to be an important indicator of lower extremity extensor muscle strength, this finding could also result from lower leg strength relative to mass (15). It has already known that a relative reduction in muscle strength observed in obese individuals from the studies comparing the relative strength of the trunk, knee and hand in obese and non-obese individuals (17). Muscle morphology determined by FTM and LBM may appear to be the major determinants of obesity-related differences in muscle strength. On the other hand, fat tissue has a great role in the secretion function of adipocytokines that have a catabolic effect on muscles by means of muscle mass and strength. Schaap et al. also speculated that these adipocytokines mediate the linkage between higher FTM and loss in muscle strength (18). Unfortunately, we did not measure lower extremity muscle strength in the current study. But the both significant negative correlation between FTM and rising index and positive correlation between LBM and rising index supported this opinion. In addition, Deforche et al. conducted a study in adolescence and indicated a lower rising index in obese boys during the STS test compared with their normal-weight counterparts (19).

The findings of the current study indicated that FTM was also positively related with COG sway velocity which is an indicator of postural balance control during functional tasks. The postural balance control during STS movement is one of the fundamental requirements to achieve the proper task. STS is commonly investigated by dividing the movement into two critical events as rising and standing phases. Rising phase starts at the onset of COG progressing forward and 
ends at the first point where COG is greater than $90 \%$ of the end COG position. Standing phase is determined by the completion of COG forward progression. Postural balance control should be maintained until the task is completed. STS movement presents a challenge for balance control and stability since this transition from a sitting to a standing position changes the base of support from three points to two points of support (20). The previous study revealed that obese adolescence had greater sway velocity during STS task, especially in standing phase. It was considered that their participants had difficulties in decelerating the forward trunk motion to rise the body based on the results of previous study (19). Sibella et al. showed that obese adults rose from the chair by limiting their forward trunk flexion and moving the feet posteriorly from their initial position compared to normal weighted adults from a biomechanical point of view (13). Together with inferior lower extremity strength (concluded from observed lower rising index) and possible kinematic deviations could have impaired postural balance control of the obese individuals with higher FTM participated in the current study.

Both FTM and LBM of the obese individuals in the current study were not related with WTT during STS. WTT is the other measured parameter in STS test since it provides insight for performance of the movement (21). This period is defined as a preparation for standing which usually occurs very quickly; the time from the seat to the arrival of the COG over the feet (10).

Therefore, WTT is an independent parameter from lower extremity strength. A WTT has been suggested to be an indicator of higher postural and directional control (22). The Deforche et al. mentioned about slower transfer time to complete multiple sit to stand tests in obese boys but they did not design their study by investigating FTM or LTM of their subjects (19). We can conclude that obese individuals in this study performed WTT during STS task regardless of their fat or lean body mass.

The comparison of our results with the limited number of studies related with the obesity and STS task is difficult because of methodological differences the studies had. For instance, Deforche et al used the Balance Master system to test STS task but the characteristics of their subjects were totally different from our subjects. Sibella et al. investigated STS task in obese adults but they performed 3D motion analysis to reveal differences in kinetic and kinematic variables during STS task. They also compare the data of obese adults with non-obese ones. Furthermore, presentations of the results differ largely. Up to date, the current study is the only one that investigated what extent FTM and LBM were associated with movement strategies of STS task in individuals with obesity. It can be concluded that there is limited information regarding the impact of FTM and LBM on functional tasks in obesity, despite its considerable importance for participating in any kind of physical activity. Further studies are warranted on these topics.
STS task was chosen due to its high repeatability in daily living and its property as a being prerequisite activity for participating in any physical performance. Our findings may be useful to develop proper interventions to improve obese individual's performance during functional tasks such as changing posture from sitting to standing. Also, the results of this study promote the literature as a research used an objective method to assess balance and body composition. Our study has cross sectional design which allows to look at FTM and LBM in relation to STS skills only at one point in time. This limitation caused researchers were not able to determine at the time order of changes in the STS skills of individuals with obesity. Further research needs to find out whether training on FTM and LBM will improve STS skills of individuals with obesity in the longitudinal designs.

\section{CONCLUSION}

This study highlights yet other factors, such as FTM and LBM, that may influence the ability of obese individuals to perform STS task in their daily routine. FTM and LBM measured in the current study can explain the significant percent of the variation in rising index during STS task, meaning a decrease in FTM and an increase in LBM provided a support to rise up during STS. Moreover, it was revealed that an increase in FTM deteriorated the postural stability. Minimizing the FTM and optimizing the LBM together may help to improve rising index and postural balance skills during STS task. Improving lean tissue mass and decreasing fat tissue mass would be an effective strategy to improve STS in obese population to increase their agility and could encourage physical activity participation as well.

\section{REFERENCES}

[1] WHO. Obesity and overweight. 2020 April 1. Available from: URL: https://www.who.int/news-room/fact-sheets/detail/ obesity-and-overweight.

[2] Finkelstein EA, Khavjou OA, Thompson H, Trogdon JG, Pan L, Sherry B, Dietz, W. Obesity and severe obesity forecasts through 2030. American journal of preventive medicine 2012; 42(6): 563-570.

[3] Wearing SC, Hennig EM, Byrne NM, Steele JR, Hills AP. The biomechanics of restricted movement in adult obesity. Obes Rev 2006; 7(1):13-24.

[4] Kejonen P, Kauranen K, Vanharanta H. The relationship between anthropometric factors and body-balancing movements in postural balance. Arch Phys Med Rehabil 2003; 84:17-22.

[5] Ledin T. Odkvist LM. Effects of increased inertial load in dynamic and randomized perturbed posturography. Acta Otolaryngol 1993; 113:249-52.

[6] McGraw B, McClenaghan BA, Williams HG, Dickerson J. Gait and postural stability in obese and nonobese prepubertal boys. Arch Phys Med Rehabil 2000; 81:484-9.

[7] Zoico E, Di Francesco V, Guralnik JM, Mazzali G, Bortolani A, Guariento S, Sergi G, Bosello O, Zamboni M. Physical disability and muscular strength in relation to obesity and different body composition indexes in a sample of healthy elderly women. Int J Obes 2004; 28(2): 234-241. 
[8] Lustig JR, Strauss BJG. Body Composition. Caballero B, editor. Encyclopedia of Food Sciences and Nutrition (Second Edition). USA: Academic Press; 2003.p.550-557.

[9] Dall PM, Kerr A. Frequency of sit to stand task: an observational study of free-living adults. Appl Ergon 2010; 41(1): 58-61.

[10] Özyürek S, Demirbüken I, Angın S. Altered movement strategies in sit-to-stand task in persons with transtibial amputation. Prosthet Orthot Int 2014; 38(4):303-309.

[11] Del Porto H, Pechak C, Smith D. Reed-Jones R. Biomechanical effects of obesity on balance Invited Review. Int J Exerc Sci 2012; 5(4): 301-320.

[12] Deforche BI, Hills AP, Worringham CJ, Davies PS, Murphy AJ, Bouckaert JJ, De Bourdeaudhuij IM. Balance and postural skills in normal-weight and overweight prepubertal boys. Int J Pediatr Obes 2009; 4(3):175-182.

[13] Sibella F, Galli M, Romei M, Montesano A, Crivellini M. Biomechanical analysis of sit-to-stand movement in normal and obese subjects. Clin Biomech 2003; 18: 745-750.

[14] Galli M, Crivellini M, Sibella F, Montesano A, Bertocco P, Parisio C. Sit-to-stand movement analysis in obese subjects. Int J Obes Relat Metab Disord 2000; 24: 1488-1492.

[15] Neurocom International, Inc Balance master system operator's manual (version 8.1). Clackamas, OR: Neurocom International, Inc., 2003.

[16] Dall PM, Kerr A. Frequency of sit to stand task: an observational study of free-living adults. Appl Ergon 2010; 41(1):58-61.

[17] Hulens M, Vansant G, Lysens R, Claessens AL, Muls E, Brumagne S. Study of differences in peripheral muscle strength of lean versus obese women: an allometric approach. Int J Obes Relat Metab Disord 2001; 25: 676-681.
[18] Schaap LA, Pluijm SM, Deeg DJ, Visser M. Inflammatory markers and loss of muscle mass (sarcopenia) and strength, Am J Med 2006; 119:9-17.

[19] Deforche BI, Hills AP, Worringham CJ, Davies PS, Murphy AJ, Bouckaert JJ, De Bourdeaudhuij IM. Balance and postural skills in normal-weight and overweight prepubertal boys. Int J Pediatr Obes 2009; 4(3):175-182.

[20] Mourey F, Pozzo T, Rouhier-Marcer I, Didier JP. A kinematic comparison between elderly and young subjects standing up from and sitting down in a chair. Age Ageing 1998; 27(2):13746.

[21] Ko MC, Wu LS, Lee S, Wang CC, Lee PF, Tseng CY, Ho CC. Wholebody vibration training improves balance control and sit-tostand performance among middle-aged and older adults: a pilot randomized controlled trial. Eur Rev Aging Phys Act 2017; 14(1):11.

[22] Lee CC, Wang RY, Yang YR. Correlations among balance and mobility measures for patients with stroke. FJPT 2003; 28(3):139-46.

[23] Clayton, J.A. and C. Tannenbaum, Reporting Sex, Gender, or Both in Clinical Research? JAMA, 2016. 316(18): p. 1863-1864.

[24] Team RC and R: A language and environment for statistical computing. R Foundation for Statistical Computing 2013. Available from: URL: http://www.R-project.org/.

[25] Völgyi E, Tylavsky FA, Lyytikäinen A, Suominen H, Alén M, Cheng . Assessing body composition with DXA and bioimpedance: effects of obesity, physical activity, and age. Obes 2008; 16(3):700-705.

How to cite this article: Timurtas E, Avci EE, Demirbuken I, Akgun I, Sertbas Y, Polat MG. The Relationship Between Fat Tissue \& Lean Body Mass and Sit to Stand Task in Obese Individuals. Clin Exp Health Sci 2021; 11: 291-295. DOI: 10.33808/ clinexphealthsci.776284 